\title{
La transferencia de los proyectos de innovación docente: un estudio sobre su capacidad de transformar la enseñanza y el aprendizaje
}

\author{
Mònica Feixas \\ Zurich University of Teacher Education. Suiza. \\ monica.feixas@phzh.ch \\ María-Jesús Martínez-Usarralde \\ Universidad de Valencia. España. \\ m.jesus.martinez@uv.es
}

Recibido: 29/4/2021

Aceptado: 9/12/2021

Publicado: 31/1/2022

\section{Resumen}

El artículo reflexiona sobre la capacidad de los proyectos de innovación docente financiados en convocatorias universitarias para transformar las prácticas educativas, la cultura de aprendizaje de los equipos pedagógicos, el desarrollo de titulaciones y el avance de la didáctica de la disciplina (Scholarship of Teaching and Learning - SoTL). Articula, como estudio de caso, una investigación sobre la evaluación de la transferencia de los proyectos de innovación docente en la Universidad de Valencia (UV). Se utiliza un enfoque metodológico mixto basado en un cuestionario elaborado ad hoc, unas entrevistas y un grupo de discusión. Los resultados ofrecen evidencias de innovaciones interdisciplinarias y con conceptos disruptivos; en menor medida en espacios atrevidos, basados en la investigación y con estudiantes como protagonistas. Su impacto ha sido evidente en la mejora de la experiencia del estudiantado y en la cultura del equipo docente, pero menos decidido en la titulación, en el avance de la didáctica de la disciplina o en el desarrollo institucional. Tras evidenciar cuestiones críticas que afectan a la gestión, a las dinámicas y al apoyo a la innovación, las conclusiones sugieren promover convocatorias de actualización docente que apoyen a la estrategia educativa universitaria de mejora del aprendizaje y de impulso al desarrollo académico docente en la UV: con el estudiante como cocreador, basadas en las necesidades de la titulación y la didáctica disciplinaria, contabilizando el coste real de la innovación para darle la visibilidad necesaria y con el apoyo pedagógico suficiente para que contribuyan a promover el avance de SoTL.

Palabras clave: educación superior; educación transformadora; innovación docente; proyectos de innovación; transferencia de aprendizaje

Resum. La transferència dels projectes d'innovació docent: un estudi sobre la seva capacitat de transformar l'ensenyança i l'aprenentatge

L'article reflexiona sobre la capacitat dels projectes d'innovació docent finançats en convocatòries universitàries per transformar les pràctiques educatives, la cultura d'aprenentatge dels equips pedagògics, desenvolupar titulacions i avançar en la didàctica de la disciplina o la Scholarship of Teaching and Learning (SoTL). Articula, com a estudi de cas, una 
investigació sobre l'avaluació de la transferència dels projectes d'innovació a la Universitat de València (UV) i s'hi utilitza un enfocament metodològic mixt basat en un qüestionari elaborat ad hoc, unes entrevistes i un grup de discussió. Els resultats mostren evidències d'innovacions interdisciplinàries i amb conceptes disruptius; en menor mesura, en espais atrevits, basats en la recerca o amb els estudiants com a protagonistes. L'impacte ha estat evident en la millora de l'experiència dels estudiants en l'assignatura i la cultura de l'equip. Menys decidit ha estat l'impacte en la titulació, en l'avenç de la didàctica de la disciplina o en el desenvolupament institucional. Després d'evidenciar qüestions crítiques que afecten la gestió, les dinàmiques i el suport a la innovació, les conclusions suggereixen promoure convocatòries d'innovació pedagògica que donin suport a l'estratègia docent universitària de millora de l'aprenentatge i d'impuls al desenvolupament acadèmic docent a la UV: amb l'estudiant com a cocreador, basades en les necessitats de la titulació i la didàctica disciplinària, comptabilitzant el cost real de la innovació per donar-li la visibilitat necessària $\mathrm{i}$ amb prou suport pedagògic perquè contribueixin a promoure l'avenç de SoTL.

Paraules clau: educació superior; educació transformadora; innovació docent; projectes d'innovació; transferència d'aprenentatge

\section{Abstract. Transfer of educational innovation projects: A study of their capacity to transform teaching and learning}

The article analyses the capacity of university-funded educational innovation projects to transform teaching practices, the learning culture of teaching teams, the development of study programmes and the advancement of the Scholarship of Teaching and Learning (SoTL). It describes, as a case study, a research project which aims to evaluate transfer in educational innovation projects at the University of Valencia (UV). A mixed methodological approach is used, based on an original questionnaire, interviews and a focus group. Results provide evidence of interdisciplinary and disruptive innovations, and to a lesser extent in unusual spaces, research-based or with students as protagonists. Its impact has been evident in improving the student experience and the culture of the teaching team. Less conclusive has been its impact on the study programme, on the advancement of SoTL and on institutional development. After assessing critical issues regarding management, dynamics and support for innovation, conclusions suggest promoting strategies to improve learning and boosting academic teaching development at UV: with students as co-creators, based on the needs of the study programme and of the disciplinary didactics, accounting for the real cost of innovation to give it the necessary visibility, and with sufficient pedagogical support to contribute to the advancement of SoTL.

Keywords: higher education; transformative education; teaching innovation; innovation projects; transfer of learning; transformation

\section{Sumario}

\section{Introducción}

2. Transformar la calidad de la enseñanza mediante los proyectos de innovación

3. Evaluación del impacto y transferencia de la innovación docente: el caso de la Universidad de Valencia
4. Discusión y conclusiones

Referencias bibliográficas 


\section{Introducción}

La innovación educativa, sea iniciada por el profesorado, por la institución o por una emergencia social, responde a una actitud constructiva de desarrollo de nuevas ideas y propuestas, efectuadas habitualmente de manera colectiva, para solucionar situaciones problemáticas de la práctica. Conocer de qué forma la innovación transforma no solo los procesos de enseñanza y aprendizaje, sino también la cultura de los equipos docentes y la universidad, así como el conocimiento disciplinario, contribuye a desarrollar la calidad docente.

Las universidades han estado apostando desde hace décadas por una educación transformadora y de calidad, entre otras, a través de iniciativas que estimulan el desarrollo de proyectos didácticos, con el objetivo de actualizar los formatos de enseñanza, aprendizaje y evaluación, además de introducir nuevas prácticas (como, por ejemplo, los rediseños híbridos de cursos, el aprendizaje-servicio o la clase invertida), mejorar el compromiso y la participación del estudiante, fomentar la colaboración entre titulaciones, etc., de acuerdo con las líneas estratégicas universitarias. Estos proyectos adquieren la forma de convocatorias internas que apelan al profesorado a implementar una de estas ideas en un periodo de uno o dos años, con un pequeño apoyo económico y bajo la iniciativa y la gestión de un servicio central específico, dependiente de un vicerrectorado. Su objetivo es incentivar la innovación educativa para que no solo revierta en una mejora de la formación estudiantil (De la Torre, Gómez-Sancho y Pérez-Esparrells, 2017), sino también para que tenga un impacto sistemático en la organización (Schophuizen y Kalz, 2020) y en el avance de la didáctica disciplinaria o SoTL (Feixas, 2019b).

Los tiempos han cambiado radicalmente (y la pandemia ha puesto a prueba a los docentes de modo extraordinario), las estrategias de hace algunas décadas sirven menos a la nueva realidad y la investigación educativa proporciona nuevas claves para la transformación del aprendizaje. Existen numerosos estudios sobre el análisis de las innovaciones universitarias (Tomàs et al., 2010), con indicadores de calidad de la innovación docente (López, Hinojosa y Sánchez; 2014) o sobre los retos organizativos en la implementación de cambios (Schophuizen y Kalz, 2020), pero existen pocos que busquen determinar el impacto de las innovaciones en las concepciones y en las prácticas del profesorado, en el desarrollo de su carrera docente, en la cultura del equipo educativo y en el aprendizaje estudiantil, así como en el desarrollo de las titulaciones, de la facultad o de la universidad y en la didáctica disciplinaria específica. Estudios que han de servir de referencia a las universidades para orientar la toma de decisiones sobre sus políticas de desarrollo docente e innovación educativa (European Commission-EACEA-Eurydice, 2018; Fernández y Madinabeitia, 2020).

A menudo el inicio de la innovación tiene lugar en proyectos a pequeña escala con recursos limitados impulsados por el entusiasmo y el compromiso de los individuos. Sin un apoyo específico, es difícil crear espacios para la exploración y la innovación en el funcionamiento normal de la docencia. El 
hecho de tener convocatorias institucionales con una financiación adicional contribuye a facilitar que el profesorado adquiera paulatinamente el hábito de examinar la enseñanza de forma didáctica y sistemática y publique resultados en revistas especializadas, abriendo una senda en la docencia basada en evidencias (Fernández y Madinabeitia, 2020). Sin embargo, el hecho de ser a pequeña escala pone en riesgo la continuidad más allá de la financiación inicial o la fase piloto (Deepwell y Buckley, 2013), y limita el impacto sistemático a nivel organizativo (Schophuizen y Kalz, 2020), pedagógico o el avance de la didáctica de la disciplina (Feixas, 2019b). Fernández y Madinabeitia (2020) ponen de relieve que

[...] el número de universidades que cuentan con iniciativas que tienen mayor impacto y que contribuyen de forma efectiva al fortalecimiento de la docencia desciende sensiblemente, y crece el número de universidades que opta por iniciativas de carácter más puntual y ligero pero sin horizontes claros de desarrollo. (p. 19)

Esta información debería hacernos plantear dicho cambio de dirección de las políticas educativas universitarias y cómo incide en la calidad y en el desarrollo estudiantil y académico docente (Paricio, Fernández-March y Fernández-Fernández, 2019).

Nuestro artículo debate acerca de las convocatorias de innovación docente, su capacidad de transformar las prácticas educativas y su impacto más allá del aula (en la titulación, en la universidad o incluso en la didáctica disciplinaria), a través de la Scholarship of Teaching and Learning (SoTL). Toma especial relevancia la consideración de los conceptos de transformación docente y transferencia de la innovación. ¿ Son los proyectos de innovación realmente transformadores de la práctica?, ¿contribuyen a facilitar el desarrollo de SoTL?, ¿adoptan los docentes las nuevas innovaciones?, ¿cómo se visibiliza su impacto?

Para responder a estas preguntas nos centramos en un caso concreto, el estudio realizado por las autoras en la Universidad de Valencia (Feixas et al., 2016), para el que analizamos de nuevo sus datos desde el punto de vista de la capacidad transformadora de los proyectos. Sus resultados sugieren algunas respuestas a las preguntas posteriores.

\section{Transformar la calidad de la enseñanza mediante los proyectos de innovación}

Evaluar la calidad de los procesos y de los resultados de la innovación no resulta sencillo. Requiere examinar numerosos criterios de calidad (Hinojosa y Sánchez, 2014) y dimensiones complejas (Tomàs et al., 2010). Los estudios existentes indican que los proyectos de innovación a pequeña escala están cambiando positivamente la percepción de la importancia de la docencia universitaria y se ha demostrado que mejoran el aprendizaje y la enseñanza (Deepwell y Buckley, 2013; Feixas, Martínez-Usarralde y López-Martín, 2017). Deepwell y Buckley (2013) reconocen que el entusiasmo del individuo cons- 
tituye la clave del éxito de la innovación y del cambio a largo plazo, y que la financiación a pequeña escala tiene más probabilidades de éxito cuando el apoyo institucional «de abajo a arriba» coincide con el entusiasmo «de arriba a abajo» de los académicos financiados. Dichos resultados coinciden con la teoría de la difusión de Rogers (2009), para quien las percepciones de los adoptantes sobre las características de la innovación son más importantes que las medidas objetivas de las mismas. No obstante, desde nuestro punto de vista (Feixas et al., 2017), es necesario determinar el papel de más elementos, además del entusiasmo de la persona responsable y su equipo, porque, para asegurar la eficacia, entre otros se requiere un contexto de apoyo constante y alineado desde la institución.

Un factor determinante en el estudio sobre el impacto de las innovaciones docentes es el reconocimiento de su capacidad transformadora de la práctica educativa. Pocos estudios analizan las innovaciones docentes desde la perspectiva del contenido de los proyectos de innovación y la orientación disruptiva de su temática y de su enfoque pedagógico. Denominamos aprendizaje transformador (Escala, 2018) al que se da cuando la docencia procura que las soluciones a problemas científicos trasciendan los límites disciplinarios. Esto es, cuando otras disciplinas u otros actores participan en la formulación de los problemas y en su solución (aspectos interdisciplinarios y transdisciplinarios); la formulación de problemas y de soluciones se basa en evidencias (aprendizaje orientado a la investigación); existen propuestas codesarrolladas con el alumnado (docentes y estudiantes en cooperación); el diseño de planes de estudio, de módulos y de cursos o unidades de aprendizaje ofrecen la posibilidad de buscar enfoques alternativos a los problemas (espacios transformadores) y el alumnado experimenta la educación no solo como la adquisición de conocimientos, sino también como el desarrollo de su capacidad para reconocer conexiones y tomar decisiones responsables con otras personas (aplicación de conocimientos transformadores) (Colomer et al., 2020; Feixas, 2019a).

\section{Evaluación del impacto y transferencia de la innovación docente: el caso de la Universidad de Valencia}

Durante los años 2016-2018, se realizó en la Universidad de Valencia, por encargo del Centro Manuel Sanchis Guarner y del Vicerrectorado de Políticas de Formación y Calidad Educativa, una investigación que perseguía conocer el impacto de proyectos de innovación docente financiados en la última convocatoria (Feixas et al., 2016). Esta investigación, publicada (Feixas et al., 2017) y resumida a continuación, se presenta como estudio de caso (Wassermann, 1999). Los datos arrojados de la misma se han reanalizado para examinar los proyectos desde una nueva perspectiva: su capacidad de transformación docente. 


\subsection{Caracterización del estudio realizado}

La investigación pretendía comprender los factores que condicionaban el impacto de la innovación en la enseñanza y aprendizaje en la universidad. El estudio partía de una revisión teórica de los principales modelos sobre la aceptación, la difusión, la transferencia y la evaluación del impacto de las innovaciones docentes en el contexto de la educación superior. Por un lado, se consideraron los aspectos subjetivos y contextuales sugeridos por modelos de aceptación (Ajzen, 1991), de difusión de la innovación (Rogers, 2009) y la innovación tecnológica (Venkatesh et al., 2003) para conocer el modo en que individuos y grupos docentes adoptaban, implementaban y transferían una innovación educativa. Por otro, se estudiaron las dimensiones de calidad en las innovaciones (López, Hinojosa y Sánchez, 2014; Tomàs, Castro y Feixas, 2010) y, finalmente, se tomó como referencia el estudio sobre los factores de transferencia de la formación docente universitaria (Feixas et al., 2013).

Los conceptos transferencia e impacto se entendían de la forma siguiente: el primero, como la aplicabilidad de lo aprendido al puesto de trabajo y el segundo incluía esta capacidad de transferencia en busca de la mejora organizacional e institucional y la consolidación de nuevos conocimientos. La clave se situaba en cómo esos conocimientos y esas nuevas competencias conducían y provocaban cambios institucionales dirigidos a la mejora continua.

Del modelo teórico se derivaron cinco categorías de factores principales que incidían en la adopción, implementación y evaluación de la transferencia y del impacto de la innovación (figura 1): factores del diseño de la innovación, del docente, del grupo de innovación, del contexto y del estudiantado.

Para obtener evidencias se empleó un enfoque metodológico mixto de tipo transversal combinando instrumentos de recogida de datos cuantitativos y cualitativos en un único momento en el tiempo. El cuestionario sobre factores de transferencia de la innovación docente (CFTID), cuyos ítems fueron previamente validados a partir de criterios de univocidad, pertinencia e importancia por diez expertos (responsables de los programas de formación e innovación docente y coordinadores de proyectos), tiene una elevada consistencia interna $(\alpha=0,953)$. Se eliminaron dos ítems para asegurar la máxima consistencia interna de las escalas y se revisaron los factores «recursos personales para innovar» y «expectativas de esfuerzo». El cuestionario resultó en 62 ítems (en escala Likert de 5 puntos), agrupados en los 14 factores de la figura 1, junto con 8 ítems de perfil. Esta información y los detalles acerca de los valores de consistencia interna, resultados del proceso de validación y la caracterización de cada uno de los factores se encuentran publicados en Feixas et al. (2017).

Simultáneamente se realizan ocho entrevistas individualizadas en profundidad a una selección de coordinadores de grupos de innovación (según disponibilidad) para conocer sus experiencias de desarrollo e implementación en los proyectos aprobados. Las cuestiones específicas respecto al impacto de la innovación docente incluyen: mejoras en el aprendizaje estudiantil; en la cultura de enseñanza y aprendizaje entre los profesores del grupo de innovación; 
Figura 1. Relación de factores condicionantes del impacto de la innovación

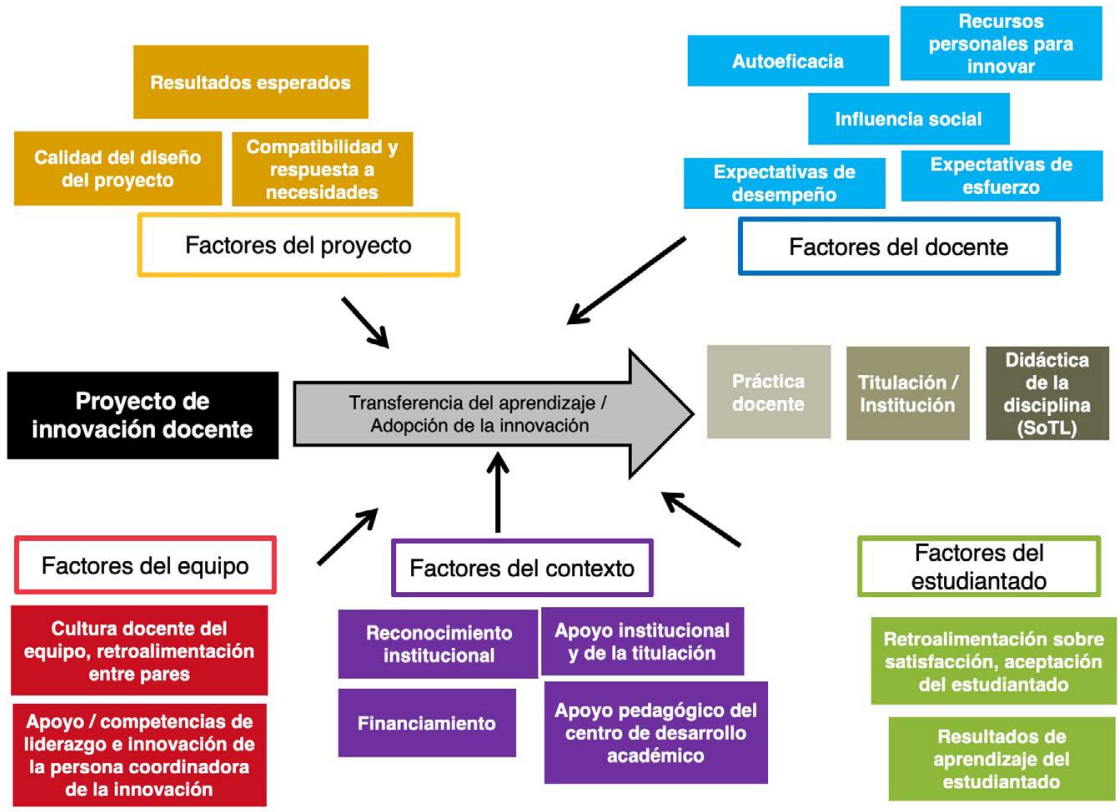

Fuente: elaboración propia.

en la cultura de enseñanza y aprendizaje de la titulación, de la facultad y de la universidad, y factores influyentes para que el proyecto tenga un impacto duradero en las competencias y en la práctica docente del profesorado en su disciplina. El análisis del contenido de las entrevistas se realiza con ATLAS.ti 8 , del que resulta un listado jerarquizado de nueve códigos.

El grupo de discusión, formado por 8 personas (expertos en educación, miembros de la unidad de formación e innovación docente, así como coordinadores de proyectos), profundiza acerca de los resultados obtenidos y se le pide que identifique posibles propuestas para mejorar la transferencia.

De un total de 453 personas participantes en los 73 proyectos de innovación financiados (36 proyectos del programa de apoyo al desarrollo de TIC y 37 del programa de apoyo a la implementación de metodologías activas, trabajo y estudio colaborativo y estrategias de evaluación innovadoras), se obtuvieron 209 respuestas del cuestionario. Sus datos se recogieron inicialmente en un archivo Excel y después se transformaron y se analizaron con el programa estadístico SPSS v.17 Inc.

Los análisis incluyeron (Feixas et al., 2017): a) propiedades psicométricas del instrumento — análisis factorial exploratorio y análisis de fiabilidad-; b) estadísticos descriptivos; $c$ ) comparación de medias — con la ayuda de estadísticos inferenciales para muestras no paramétricas como son U de Mann- 
Whitney y H de Kruskal-Wallis, calculando también el tamaño del efecto-, y d) análisis correlacionales - utilizando el estadístico $r$ de Pearson.

11 factores de 14 (a excepción de «Institucionalización de la innovación en la cultura de enseñanza y aprendizaje», «Retroalimentación y resultados de aprendizaje en el estudiante» $\mathrm{y}$ «Condiciones facilitadoras») obtienen puntuaciones entre 4 y 5 , lo que denota una percepción muy positiva de los mismos, actuando como facilitadores fuertes del impacto de la innovación docente. El factor «Retroalimentación y resultados de aprendizaje en el estudiante» obtiene un valor entre 3 y 4 , lo que indica un facilitador débil de la transferencia. Y los factores "Condiciones facilitadoras» e "Institucionalización de la innovación en la cultura de enseñanza y aprendizaje», con valores entre 2 y 3 , actúan como posibles barreras para el impacto.

La figura 2 muestra los resultados medios de todos los factores:

Figura 2. Factores facilitadores del impacto de la innovación docente

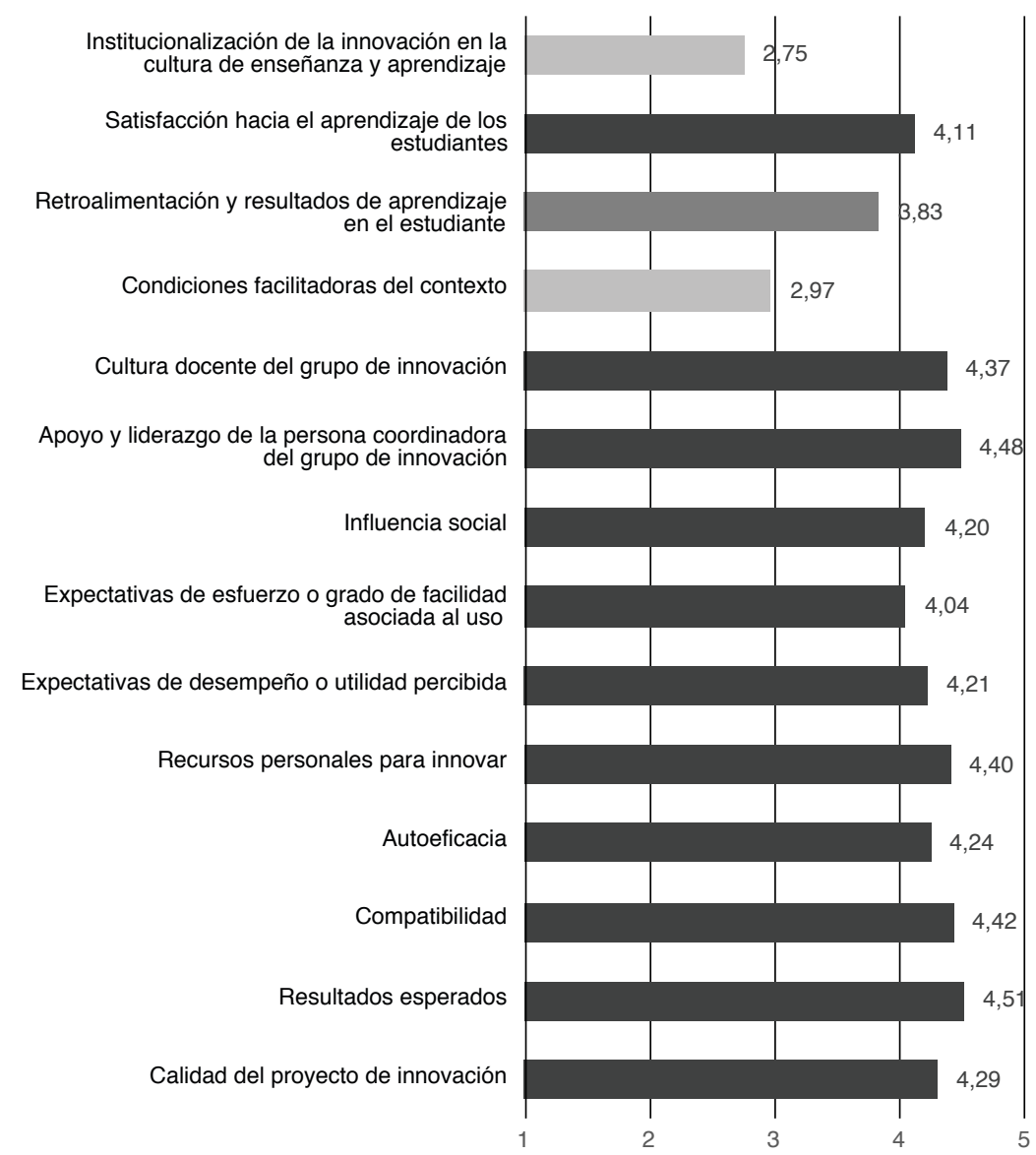

Fuente: elaboración propia. 
Los resultados de las entrevistas y el focus group subrayaron que los fondos ejercieron:

- Un gran impacto en la enseñanza, ya que el diseño del proyecto era capaz de medir claramente los resultados esperados.

- Un impacto considerable en el desarrollo profesional individual, porque los profesores están motivados intrínsecamente por el compromiso personal, y la inversión de tiempo es gratificante, ya que proporciona ganancias a largo plazo.

- Un impacto menor en la disciplina y en el programa de estudios, pero cierto beneficio en el desarrollo de algunos conocimientos disciplinarios didácticos.

- Un impacto significativo en la cultura de enseñanza del equipo, porque fortaleció la cooperación entre pares y ofreció un espacio para explorar alternativas conceptuales, mejorar las habilidades y servirse de nuevas herramientas.

- Un impacto limitado en la institucionalización de la innovación, porque la financiación y el apoyo pedagógico y técnico en la implementación no fueron suficientes, a pesar de ser bienvenidos. También el reconocimiento de su trabajo en términos de promoción y visibilidad fue limitado.

Aunque no fue posible medir el impacto de cada innovación en el aprendizaje de los estudiantes, los encuestados manifestaron un aumento positivo de la satisfacción de los estudiantes con la adquisición de nuevos conocimientos.

\subsection{La capacidad de transformación docente en y de los proyectos de innovación}

En este apartado se examina la capacidad del profesorado y del proyecto de transformar la docencia. Para ello se han analizado de nuevo los resultados de los cuestionarios y de las entrevistas. Por un lado, se reelabora sobre los factores que tienen relación con las características del docente que implementa una innovación y, por otro, se examina la capacidad transformativa de los diseños y de los formatos de educación de acuerdo con las características presentadas en el marco teórico.

Respecto a la capacidad del profesorado de transformar su práctica, los valores obtenidos del cuestionario de la autoeficacia, los recursos personales para innovar, las expectativas de desempeño, de esfuerzo y la influencia social son superiores a $4 \mathrm{y}$, como facilitadores fuertes de la transferencia, demuestran que el profesorado:

- Cree en su iniciativa, se siente capaz, confiado y seguro ante la implementación de propuestas novedosas y puede superar obstáculos ("Autoeficacia»). Según los entrevistados, la innovación les ha servido para examinar de forma sistemática los resultados de sus prácticas y las condiciones de aprendizaje de su estudiantado, considerando como resultado que ahora son más eficaces. 
- Tiene implicación, energía y puede dedicar tiempo a implementar la innovación («Recursos personales para innovar»). La implicación, o engagement, es la motivación y el compromiso que se tiene para desarrollar las iniciativas con resultados finales exitosos. En el focus group se debate que la continuidad de un proyecto de innovación va ligada a la necesidad de que los docentes a cargo estén dispuestos a reinventar el proyecto continuamente a partir de analizar los logros y vislumbrar nuevas posibilidades de implementación.

- Cree que la innovación le facilitará la obtención de beneficios en el trabajo («Expectativas de desempeño»). En las entrevistas, los docentes expresan la utilidad de sus innovaciones en tanto que están directamente relacionadas con una mejora concreta del proceso de enseñanza-aprendizaje y porque les han permitido ofrecer a los estudiantes experiencias más contextualizadas con las que vislumbrar la aplicabilidad de lo que aprenden.

- Percibe un grado factible de facilidad de implementación de la innovación («Expectativas de esfuerzo») o baja complejidad. Tiene asimismo expectativas de que el esfuerzo realizado sea reconocido y valorado por la institución.

- Aprecia que la innovación le haya servido para mejorar su propia imagen profesional o su estatus en el contexto universitario («Influencia social»).

Desde el punto de vista de la educación transformadora (Colomer et al., 2020; Feixas, 2019a), se ha examinado en qué medida los proyectos de innovación analizados poseen las cinco características identificadas: interdisciplinariedad; aprendizaje orientado a la investigación; en colaboración con estudiantes o personas externas; en espacios alternativos, y que busquen la aplicación de conocimientos transformadores.

En concreto, la mayoría de los 37 proyectos del programa de apoyo a la implementación de metodologías activas, trabajo y estudio colaborativo y estrategias de evaluación innovadoras muestran como mínimo dos de las cinco características: aplicación de conocimientos transformadores y diseño de experiencias de aprendizaje alternativas. En este sentido, el estudiantado experimenta la educación no solo como la adquisición de conocimientos, sino también como el desarrollo de su capacidad para reconocer conexiones y tomar decisiones responsables con otros (aplicación de conocimientos transformadores). Un ejemplo tiene que ver con un proyecto que diseña herramientas que permitan trascender prácticas socialmente cuestionadas, como es el caso de la experimentación con animales.

Anteriormente utilizábamos animales para hacer esta práctica y, al grabarlo en un vídeo, ya no tenemos que utilizarlos más. La idea era reducir al mínimo el número de animales vivos. Podía ayudar a esto hacer grabaciones en vídeo de lo que habitualmente ven en el laboratorio en vivo [...]. Entonces, la idea era, primero, darles la posibilidad de verlo in situ con el vídeo y, lo segundo, no utilizar más animales para las prácticas de docencia. (E6) 
Otro ejemplo también identifica claramente cambios en las formas de enseñanza que han redundado en mejoras visibles de las habilidades del alumnado mediante nuevas formas de acercarse al estudio del diseño y del dibujo.

Lo que hemos hecho este año ha sido cambiar la forma de explicar. Nosotros trabajamos con sacar vistas de piezas en dibujo técnico. Hasta ahora dábamos unas fotocopias de Anaya donde había las vistas y tenían que dibujar en isométrico o en axonométrico y tenías que sacar las vistas. Ahora ellos primero lo diseñan en 3D y luego sacan las vistas. Es mucho más rápido para la mejora de las habilidades espaciales, al contrario del método tradicional. (E8)

Desarrollar nuevas experiencias de aprendizaje implica involucrarse en un ejercicio de creatividad para explorar alternativas que enriquezcan los ambientes de aprendizaje:

Normalmente hay anfiteatros tipo americano en que el aula misma tiene un personal y al lado está el gabinete donde están todos los materiales. El profesorado tiene que ir allí y llevarlo al aula, y devolverlo. Entonces la versión original es mucho más sofisticada y nosotros la hemos transformado en una versión transportable al aula. (E2)

Un considerable grupo de proyectos promueve experiencias docentes en espacios transformadores no convencionales. Nos referimos a la presencialidad en los espacios virtuales, y aquí encontramos muchos proyectos del programa de fomento de las TIC, en concreto aquellos plenamente en línea, como los que desarrollan los MOOC. O bien aquellos proyectos que tienen lugar en espacios físicos alejados de la tradicional aula universitaria.

Una parte de los proyectos están formados por docentes de distintas disciplinas (interdisciplinariedad y transdisciplinariedad). El desarrollo de las innovaciones por grupos interdisciplinares motiva el interés por debatir acerca de diferentes realidades y ofrece posibilidades de aprendizaje conjunto, aspectos que además fortalecen los equipos docentes.

Nos hemos juntado gente que éramos complementaria, multidisciplinar, no nos hemos cerrado a trabajar en ámbitos sino que lo hemos abierto mucho para podernos juntar con gente de otras titulaciones. Yo creo que eso ha sido lo que ha favorecido que sigamos. (E8)

En menor medida encontramos proyectos donde la formulación de problemas y soluciones se basa en evidencias (aprendizaje orientado a la investigación) o donde los estudiantes pueden incorporarse parcial o totalmente a un análisis basado en métodos científicos bajo la supervisión del profesor. Algunos demuestran su capacidad investigativa al expresar que difunden los resultados en foros científicos:

Esto es un proyecto que lleva bastantes años y hemos hecho varias cosas. Este año vamos a presentar un vídeo a Ciencia en Acción, llevándolo a un foro un poco más amplio. Como es una cosa completamente exportable, porque son 
vídeos y simulaciones, podrían ser de utilidad en otras universidades. Por eso queremos darle difusión: un par de reuniones de la Real Sociedad Española de Física, la publicación en la revista de la Universidad y ahora lo vamos a enviar a Ciencia en Acción. (E4)

En la misma línea, encontramos pocos proyectos que consideran al estudiantado como cocreador de conocimiento y solo un par que trabajan propuestas codesarrolladas con entidades externas como el gobierno local u organizaciones no gubernamentales.

El diseño de planes de estudio, módulos y cursos o unidades de aprendizaje que ofrecen la posibilidad de trabajar en espacios singulares son también limitados. Un ejemplo de ello lo constituye el proyecto sobre rutas históricas y lingüísticas, que asimismo posee todos los demás ingredientes de un programa de aprendizaje transformador: el diseño y la formulación de problemas y soluciones son codesarrollados con el alumnado, se trabaja desde la indagación y la investigación (estudio conceptual, descubrimiento, recogida de evidencias y reflexión), es interdisciplinario (historia, lengua y literatura catalana), se da en espacios distintos (fuera del campus) y se presentan experiencias totalmente alternativas:

La gente que ha preparado las rutas, las han probado. Han ido a El Puig un domingo y han comprobado que todo es exacto. Son propuestas para todas las universidades de la Xarxa, los alumnos hacen rutas literarias asociadas a las asignaturas. Cuando el alumno ha salido de ruta con el profesor y ha compartido, nosotros notamos que el aprendizaje ha mejorado porque lo hemos contextualizado, lo hemos hecho real. Nuestro componente de innovación es el experimentar la emoción de un paisaje, de salir fuera del aula, de compartir unas horas de convivencia, o un día con los propios alumnos. (E1)

\section{Discusión y conclusiones}

Las convocatorias que promueven los proyectos de innovación brindan grandes oportunidades a la comunidad universitaria. Por un lado, ofrecen apoyo al personal docente que desea llevar a cabo un concepto transformador, que quiere mejorar, cambiar o evaluar la eficacia de una intervención; permiten avanzar en el trabajo cooperativo en equipos interdisciplinarios, y pueden contribuir a utilizar un enfoque académico basado en la investigación docente (SoTL). Sin embargo, los temas son a menudo de interés personal y no institucional, el dinero no siempre alcanza los propósitos del proyecto y el impacto en la institución es limitado (Deepwell y Buckley, 2013).

Tras años de evaluar proyectos de innovación docente, la experiencia y los estudios acerca de la adopción (Rogers, 2009, Venkatesh et al., 2003) y del impacto (Feixas et al., 2013; Feixas, 2019b) nos han enseñado dónde reside la calidad de la innovación. Existen una serie de factores relacionados con el diseño del proyecto, el docente y el grupo de innovación, las condiciones del contexto y la respuesta del estudiantado que inciden en mayor o menor medida en la adopción, la implementación y la evaluación de la transferencia y el 
impacto de la innovación. Analizar hasta qué punto los proyectos de innovación tienen la capacidad de revertir en una mejora de la práctica docente, la experiencia de aprendizaje del estudiantado, el desarrollo institucional y el avance de la didáctica disciplinaria es posible desde el estudio de los factores condicionantes y del tipo de proyecto que se desarrolla.

Este artículo pone en evidencia que no todos los proyectos de innovación analizados tienen la misma capacidad transformadora. La mayoría se caracteriza principalmente por utilizar nuevas metodologías mediadas por las TIC (blog, clase invertida, MOOC, etc.), por trabajar el conocimiento de forma integrada desde distintas disciplinas y algunos por proponer espacios alternativos al aula. Pocos incluyen al colectivo estudiantil como copartícipe del programa o como posible cocreador de contenido (Healey y Healey, 2019) y todavía menos fomentan la investigación.

La transferencia y el impacto potencial de los proyectos de innovación son positivos dados los altos valores alcanzados en el cuestionario de factores y resultan evidentes en el cambio de las prácticas docentes gracias al análisis de las entrevistas (Feixas et al., 2017). Concretamente, los proyectos del estudio han desarrollado habilidades docentes y han supuesto una gran motivación y satisfacción para el profesorado, además de mejoras en los aprendizajes de los estudiantes. Además, el alumnado valora positivamente las innovaciones docentes, lo que constituye un aspecto motivador para el profesorado, porque evidencian mejoras en el rendimiento, la autorregulación y el desarrollo de competencias transversales. Tomar más en cuenta al estudiantado resulta imprescindible, especialmente su implicación en el proceso de diseño de la innovación.

En general, los proyectos analizados responden menos a necesidades de titulaciones o de la institución y más a un interés individual o de un colectivo docente. Es por esta razón que los proyectos promueven solo parcialmente el desarrollo académico docente, el disciplinario, o suponen ligeros avances en SoTL.

Los participantes reclaman mayor reconocimiento institucional y apoyo técnico. Por una parte, resulta indudablemente positivo que las instituciones reconozcan y fomenten el trabajo realizado por el profesorado implicado en procesos de innovación y que tengan el efecto de ser computados como mérito en procesos de acreditación y estabilización. Por otra, las convocatorias planteadas deberían ofrecer apoyo pedagógico y de la titulación para lograr un avance en el conocimiento disciplinario pedagógico (SoTL) y ejercer un impacto más profundo en la calidad de los programas.

Desde el punto de vista evaluativo, se evidencia la importancia de contar con herramientas que permitan visibilizar la transferencia de la innovación educativa y, de este modo, leerla en clave cuantitativa y cualitativa, obteniendo con ello una visión fiable para sus responsables. Si bien los datos del estudio de Valencia no son suficientes, ya que los participantes en el estudio de campo son menos de la mitad del total de participantes en grupos de innovación de la universidad, ni concluyentes para hacer una interpretación general de las 
políticas de innovación docente de la universidad (tampoco era este el objetivo), el análisis realizado reafirma el valor de las políticas de innovación educativa e incentiva mantener sus propuestas, al tiempo que ha de incidir en aquellos factores de mejora.

La investigación realizada en un contexto característico de la tipología de convocatorias en universidades españolas nos lleva a considerar nuevas cuestiones:

- Presupuesto y equiparación a la investigación. ¿Situar la innovación docente en la universidad como fase previa a la investigación educativa o equipararla a la investigación?, ¿cómo lograr un impacto con proyectos de innovación que tienen un financiamiento muy por debajo de los proyectos de investigación?, ¿cuál es el coste real de la innovación?, ¿qué se financia?, ¿cómo se calcula el coste de un proyecto, y el de las horas del personal?

- Dedicación de los miembros del proyecto y objetivos individuales. ¿Cuál es el interés y la motivación personal para estar en el proyecto?, ¿pueden conjugarse los objetivos individuales anuales de un docente con los objetivos del proyecto de innovación?, ¿a quién se deben el docente y sus objetivos de mejora?

- La implicación real de los estudiantes. En las evaluaciones se analizan los resultados de aprendizaje del estudiantado, y a menudo se les consulta sobre sus percepciones acerca de las intervenciones innovadoras. Sin embargo, no es llamado a participar en su diseño o evaluación. ¿Es un sujeto más de la evaluación?, ¿cuál debería ser su implicación real?

Tras evidenciar cuestiones críticas que afectan a la gestión, a las dinámicas y al apoyo a la innovación, se sugiere promover convocatorias de cambio educativo que favorezcan a la estrategia docente universitaria de mejora del aprendizaje y de impulso al desarrollo académico docente, es decir, que tengan al estudiante como protagonista y que estén basadas en las necesidades de la titulación y la didáctica disciplinaria, contabilizando el coste real de la innovación para darle la visibilidad necesaria y que cuenten con el apoyo pedagógico suficiente para que contribuyan a promover el avance de SoTL.

Dado que el proceso de innovación forma parte de una transformación más amplia relacionada con las tendencias en educación superior, es inexcusable repensar los cambios y el proceso que las acompaña de forma continua y contextualizada para captar su complejidad y dar respuesta a las nuevas necesidades docentes (Fernández y Madinabeitia, 2020; Perines, 2018).

\section{Referencias bibliográficas}

Ajzen, I. (1991). The theory of planned behaviour. Organizational Behaviour and Human Decision Processes, 50(2), 179-211.

Boyer, E.L. (1990). Scholarship reconsidered: Priorities of the professoriate. Princeton, NJ: Carnegie Foundation for the Advancement of Teaching. 
Colomer, J.; Serra, T.; Cañabate, D. y Bubnys, R. (2020). Reflective learning in higher education: Active methodologies for transformative practices. Sustainability, 12(9), 3827. <https://doi.org/10.3390/su12093827>

Deepwell, F. y Buckley, C. (eds.) (2013). SEDA small grants: Celebrating the scholarship of educational development. Londres: SEDA.

Escala, M.J. (2018). Comprendiendo y promoviendo el aprendizaje transformador: Una guía para la teoría y la práctica. 3. a ed. Ciencia y Educación, 2(1), 75-76. <http://dx.doi.org/10.22206/cyed.2018.v2i1.pp75-76>

European Commission / EACEA / Eurydice (2018). The European Higher Education Area in 2018: Bologna Process Implementation Report. Luxemburgo: Publications Office of the European Union. Recuperado de <https://bit.ly/2WXhpOG >.

FeIXAS, M. (2019a). Formación e innovación docente universitaria para una educación transformadora: la investigación e indagación reflexiva como requisito. El Guiniguada. Revista de investigaciones y experiencias en Ciencias de la Educación, 28. <https://doi.org/10.20420/ElGuiniguada.2019.265>

Feixas, M. (2019b). Proyectos de innovación y premios a la calidad docente universitaria: su contribución al desarrollo institucional docente. (Innovation funds and teaching awards: contributing to the institutional development of teaching). Proceedings. 3rd EuroSoTL Conference, Universidad del País Vasco, Bilbao. Recuperado de <https:/www.ehu.eus/es/web/eurosotl-2019/berrikuntzarako-proiektuaketa-unibertsitateko-irakaskuntzaren-kalitateari-emandako-sariak>.

Feixas, M.; Duran, M.M.; Fernández, I.; Fernández, A.; García San Pedro, M.J.; Márquez, M.D.; Pineda, P.; Quesada, C.; Sabaté, S.; Tomàs, M.; Zellweger y LaGos, P. (2013). ¿Cómo medir la transferencia de la formación en Educación Superior?: el Cuestionario de Factores de Transferencia. Red Estatal de Docencia Universitaria (RED-U), 11(3), 219-248. <https://doi.org/10.4995/redu.2013.5527>

Feixas, M. (IP); Martínez-Usarralde, M. J.; Pallás, J. y López-Martín, R. (2016). Análisis de la transferencia del conocimiento en la política de formación e innovación de la Universitat de Valencia. Informe de investigación. Documento Interno. Centre de Formació i Qualitat Manuel Sanchis Guarner. Universitat de València.

Feixas, M.; Martínez-Usarralde, M. J. y López-Martín, R. (2017). Do teaching innovation projects make a difference? Assessing the impact of small-scale funding. Tertiary Education and Management, 24(4), 263-287. <https://doi.org/10.1080/13583883.2017.1417470>

Fernández, I. y Madinabeitia, A. (2020). La transformación docente de la universidad a veinte años de Bolonia: Balance y claves para un futuro por definir. Profesorado: Revista de Currículum y Formación de Profesorado, 24(1), 28-52. <https://doi.org/10.30827/profesorado.v24i2.15149>

Healey, M. y Healey, R.L. (2019). Student Engagement through Partnership: A Guide and Update to the Advance HE Framework. Recuperado de <https://www.researchgate.net/profile/Mick-Healey/publication/338096919_Students_as_Partners_ Guide_Student_Engagement_Through_Partnership_A_guide_to_the_ Advance_HE_Framework/links/5e402bf2299bf1cdb91bd14b/Students-asPartners-Guide-Student-Engagement-Through-Partnership-A-guide-to-theAdvance-HE-Framework.pdf $>$.

López, M.C.; Hinojosa, E. y Sánchez, M.D. (2014). Evaluación de la calidad de los proyectos de innovación docente universitaria. Profesorado: Revista de Currí- 
culum y Formación de Profesorado, 18(3), 377-391. Recuperado de <https://recyt. fecyt.es/index.php/profesorado/article/view/48942>.

Paricio, J.; Fernández-March, A. y Fernández-Fernández, I. (eds.) (2019). Cartografia de la buena docencia universitaria: Un marco para el desarrollo del profesorado basado en la investigación. Madrid: Narcea.

Perines, H. (2018). ¿Por qué la investigación educativa no impacta en la práctica docente? Estudios sobre Educación, 34, 9-27. $<$ https://doi.org/10.15581/004.34.9-27>

RamíreZ-Montoya, M.S. y Lugo-Ocando, J. (2020). Revisión sistemática de métodos mixtos en el marco de la innovación educativa. Comunicar, 65, 9-20. $<$ https://doi.org/10.3916/C65-2020-01>

Tomàs, M.; Bernabeu, D.; Borrell, N.; Castro, D.; Feixas, M. y Fuentes, M. (2010). Cultura innovadora en la universidad. Madrid: Octaedro.

Tomàs, M.; Castro, D. y Feixas, M. (2010). Dimensiones para el análisis de las innovaciones en la universidad. Propuesta de un modelo. Bordón. Revista De Pedagogía, 62(1), 139-151. Recuperado de <https://recyt.fecyt.es/index.php/ BORDON/article/view/29112>.

Rogers, E.M. (2009). Diffusion of innovations. Nueva York, NY: Free Press.

Schophuizen, M. y Kalz, M. (2020). Educational innovation projects in Dutch higher education: Bottom-up contextual coping to deal with organizational challenges. International Journal of Educational Technology in Higher Education, 17(36). $<$ https://doi.org/10.1186/s41239-020-00197-z>

Torre, E.M. de la; Gómez-SAncho, J.M. y Pérez-Esparrells, C. (2017). Comparing university performance by legal status: A Malmquist-type index approach for the case of the Spanish higher Education system. Tertiary Education and Management, 28(2), 1-16. <https://doi.org/10.1080/13583883.2017.1296966>

Venkatesh, V.; Morris, M.G.; Davis, G.B. y Davis, F.D. (2003). User acceptance of information technology: Toward a unified view. MIS Quarterly, 27(3), 425-478. <https://doi.org/10.2307/30036540>

Wassermann, S. (1999). El estudio de casos como método de enseñanza. Buenos Aires: Amorrortu. 